\title{
Solid Lipid Nanoparticles (SLN) and Nanostructured Lipid Carriers (NLC): Occlusive Effect and Penetration Enhancement Ability
}

\author{
R. López-García, A. Ganem-Rondero* \\ División de Estudios de Posgrado (Tecnología Farmacéutica), Facultad de Estudios Superiores Cuautitlán, \\ Universidad Nacional Autónoma de México, Cuautitlán Izcalli, Mexico \\ Email: mrosario.logar@hotmail.com, ${ }^{*}$ ganema@hotmail.com
}

Received 12 March 2015; accepted 23 May 2015; published 28 May 2015

Copyright (C) 2015 by authors and Scientific Research Publishing Inc.

This work is licensed under the Creative Commons Attribution International License (CC BY). http://creativecommons.org/licenses/by/4.0/

(c) () Open Access

\begin{abstract}
Objective: This work compares the occlusive effect and the penetration enhancement ability of solid lipid nanoparticles (SLN) and nanostructured lipid carriers (NLC), through in vitro skin. Methods: SLN and NLC were prepared by high shear homogenization and characterized by size, polydispersity index, zeta potential, morphology and physical stability. Occlusive effect was assessed by an in vitro test and by measuring TEWL using pig skin. Skin treated with the lipid carriers was visualized by SEM. A penetration test through skin, followed by tape stripping, was carried out using Nile red as a marker. Results: SLN $(200 \pm 6 \mathrm{~nm})$ and NLC $(192 \pm 11 \mathrm{~nm})$ were obtained. An occlusion factor of $36 \%-39 \%$ was observed for both systems, while a reduction in TEWL of $34.3 \%$ $\pm 14.8 \%$ and $26.2 \% \pm 6.5 \%$ was seen after treatment with SLN and NLC, respectively. SEM images showed a film formed by the lipid carriers, responsible for the occlusion observed. No differences were found between the occlusive effect produced by SLN and NLC in both tests. NLC allowed the penetration of a greater amount of Nile red than SLN: $4.7 \pm 1.3 \mu \mathrm{g}$ and $1.7 \pm 0.4 \mu \mathrm{g}$, respectively. Conclusion: Both carriers form a film on the skin, providing an occlusive effect with no differences between these two systems. The penetration of a marker (Nile red) into the stratum corneum was quite higher for NLC than for SLN, suggesting an influence of the composition of these particles on their penetration enhancing ability.
\end{abstract}

\section{Keywords}

Solid Lipid Nanoparticles, Nanostructured Lipid Carriers, Occlusive Effect, Transepidermal Water Loss, Skin Penetration

\footnotetext{
${ }^{*}$ Corresponding author.
}

How to cite this paper: López-García, R. and Ganem-Rondero, A. (2015) Solid Lipid Nanoparticles (SLN) and Nanostructured Lipid Carriers (NLC): Occlusive Effect and Penetration Enhancement Ability. Journal of Cosmetics, Dermatological Sciences and Applications, 5, 62-72. http://dx.doi.org/10.4236/jcdsa.2015.52008 


\section{Introduction}

Nanotechnology is a highly useful tool for the design of innovative solutions for many sciences including health and beauty. The understanding and handling of compounds at nanoscale have allowed the development of materials with interesting characteristics to use in cosmetic science as seen in the design of nanoparticles of different materials as carrier systems for cosmetic actives.

Solid lipid nanoparticles (SLN) were developed at the beginning of the 1990's based on the concept of solid particles, emulsions and liposomes. They are produced by exchanging the oil in an emulsion by a solid lipid, resulting in lipid nanoparticles being solid at both room and body temperature [1]. Although SLN possess several advantages, e.g., the use of physiological lipids, the avoidance of organic solvents in the preparation process, protection of sensitive molecules from the environment and controlled release characteristics, some disadvantages have been associated such as particle growth, unpredictable gelation tendency, polymorphic transitions and inherently low incorporation capacities due to the crystalline structure of the solid lipid [2]. The second generation of lipid nanoparticles, nanostructured lipid carriers (NLC) which are prepared by blending solid and liquid lipids leading to amorphous solids, arose to overcome limitations of SLN by introducing a less ordered inner structure [3].

Nanoparticles based on lipid systems are the most common type of nanoparticles studied for topical application [4]. The current focus of the SLN and NLC research is quite related towards topical and dermal application, both in pharmaceutical and cosmetic purposes. However, since NLC represent the latest innovation, several researchers preferred them over SLN as they avoid lipid recrystallization that causes expulsion of active substances as observed with SLN [5]. Nevertheless, both SLN and NLC are widely used for cosmetics, since they show many favorable features such as adhesiveness, occlusion, skin hydration, lubrication, smoothness, emolliency, skin penetration enhancement, modified release, improvement of formulation appearance providing a whitening effect and offering protection of actives against degradation [6]-[8]. These positive features of lipid nanoparticles have led to the market introduction of a number of cosmetic products including numerous actives such as coenzyme Q10, extracts, peptides, oils, fatty acids and sun blockers [9].

The effects of lipid nanoparticles on skin barrier properties have been well established. It has been reported that SLN form an invisible, occlusive film with affinity for the stratum corneum (SC), which ensures drug release for a prolonged period of time, reduces transepidermal water loss and improves skin hydration [1] [10]. Penetration enhancement ability of SLN and NLC has also been studied [11]-[13]. Moreover, even if several papers discuss the effects of SLN and NLC on skin and their performance as carrier systems, the advantages of NLC over SLN have not yet been well established [14]-[17].

The aim of this study was to evaluate and compare the performance of SLN and NLC on the skin barrier properties through the assessment of the occlusive effect they produce and their penetration enhancement ability through SC in order to determine if differences in composition modify the performance of these systems. It is expected that the results obtained contribute to the understanding of these lipid-based carriers and improving their performance as carrier systems for cosmetic actives.

\section{Materials and Methods}

Glyceryl dibehenate (Compritol ${ }^{\circledR} 888$ ATO) was a gift from Lyontec (Mexico City). Caprylic capric triglycerides were purchased from Droguería Cosmopolita (Mexico City) and poloxamer 188 (Lutrol ${ }^{\circledR}$ F-68) was obtained from BASF (Germany). Methanol was purchased from J. T. Baker, and Nile Red was obtained from Sigma-Aldrich (USA). Water was obtained from a Milli- $Q^{\circledR}$ system (Millipore ${ }^{\circledR}$, Germany). Porcine skin was obtained from pig ears collected from a local slaughterhouse immediately post-mortem and and prior to steam cleaning (the procedure followed the guidelines of The Mexican Official Standard NOM-194-SSA1-2004, related to the sanitary specifications for abbatoirs). Pig ears with no injuries and with uniform coloration were selected and stored at $-20^{\circ} \mathrm{C}$ until required.

\subsection{SLN and NLC Preparation}

SLN (composed of glyceryl dibehenate) and NLC (including glyceryl dibehenate/caprylic capric triglycerides 90:10) were prepared by high shear homogenization using an Ultra Turrax ${ }^{\circledR}$ T18 Basic (IKA ${ }^{\circledR}$, Germany). Briefly, a hot $2.5 \%$ poloxamer 188 solution $\left(90^{\circ} \mathrm{C}\right)$ was added to the melted lipid phase (solid lipid or lipids blend). This mixture was emulsified at 20,000 rpm for $5 \mathrm{~min}$ at $90^{\circ} \mathrm{C}$. The resultant emulsion was cooled down 
in a cold water bath $\left(7^{\circ} \mathrm{C}\right)$ to room temperature in order to obtain nanoparticles.

\subsection{SLN and NLC Characterization}

Particle size, polydispersity index (PI) and zeta potential were determined by photon correlation spectroscopy using a Malvern Zetasizer ${ }^{\circledR}$ Nano-ZS90 (Malvern Instruments, USA). For size and PI, the samples were diluted with distilled water to a proper mean count rate prior to the measurements, which were performed at a $90^{\circ}$ scattering angle at $25^{\circ} \mathrm{C}(\mathrm{n}=5)$. To measure zeta potential, samples were diluted with deionized water and the measurements were done considering viscosity, refraction index and dielectric constant of water at $25^{\circ} \mathrm{C}(\mathrm{n}=5)$. The morphology of the lipid nanoparticles was evaluated by means of scanning electron microscopy (SEM) using a JEOL JSM-25SII Scanning microscope (JEOL Tokyo, Japan). To prepare the sample, nanoparticle aqueous dispersion was left to dry over a coverslip and covered with gold.

\subsection{SLN and NLC Stability}

SLN and NLC formulations were stored in glass vials at $5^{\circ} \mathrm{C}, 25^{\circ} \mathrm{C}$ and $40^{\circ} \mathrm{C}$. Particle size, PI and zeta potential were measured at $8,15,30,60$ and 90 days $(\mathrm{n}=3)$, as previously described.

\subsection{Test of in Vitro Occlusive Effect}

Occlusive effect of SLN and NLC was determined using a modified in vitro occlusion test [18]. Briefly, bakers were filled with $40 \mathrm{~mL}$ of water and covered with Whatman ${ }^{\circledR}$ filter paper grade 42 (Sigma-Aldrich, USA). A sample of SLN or NLC (300 $\mu \mathrm{L}, 10 \% \mathrm{w} / \mathrm{v}$ lipid content) was spread on the filter surface, using water as reference, instead lipid carriers. Bakers were stored at $32^{\circ} \mathrm{C}$ to mimic the temperature of skin surface and weighted at 0, 6, 24 and $48 \mathrm{~h}$ to calculate water evaporation through the filter paper in terms of water loss. The occlusion factor $F$ was calculated at 6, 24 and $48 \mathrm{~h}$ using Equation (1) [18].

$$
F=\frac{R-S}{R} \times 100
$$

where $R=$ reference water loss and $S=$ sample water loss. An $F=0$ means no occlusive effect while an $F=100$ means maximum occlusiveness. At the end of the experiment, filter paper was observed by scanning electron microscopy (SEM), using a JSM-25-SII scanning electron microscope (JEOL, Tokyo, Japan) in order to visualize film formation.

\subsection{Effect of SLN and NLC on the TEWL}

Transepidermal water loss (TEWL) is the outward diffusion of water through skin [19]. Skin was excised from pig ears. Skin slides (700 $\mu \mathrm{m}$ thickness) were obtained using an Electric Dermatome (Zimmer ${ }^{\circledR}$, USA). Impaired skin was obtained removing the SC by means of tape stripping (20 tapes). Both impaired and intact pig skin samples were mounted on the receptor compartment of a Franz cell filled with phosphate buffer solution $\mathrm{pH} 7.4$ and basal TEWL value was recorded with a Tewameter ${ }^{\circledR}$ TM 210 (Courage \& Khazaka, Germany). A volume of $50 \mu \mathrm{L}(10 \% \mathrm{w} / \mathrm{v})$ of SLN or NLC dispersion was spread on the skin surface and TEWL was measured $2 \mathrm{~h}$ later. The occlusive effect was determined by the reduction of the TEWL value using Equation (2):

$$
\% \text { TEWL reduction }=\frac{B-T}{B} \times 100
$$

where $B$ is the basal TEWL value and $T$ is the TEWL value after lipid carrier treatment. SEM was used to visualize film formation on the skin. Pig skin was fixed, dehydrated and coated with gold previous observation under the microscope (JEOL JSM-25-SII, JEOL, Tokyo, Japan).

\subsection{Penetration Test through Pig Skin}

Penetration test through the SC of pig skin was carried out using SLN and NLC containing Nile red as a marker. Nile red-loaded SLN and NLC $(0.005 \% \mathrm{w} / \mathrm{w})$ were prepared as described before, incorporating the dye in the melted lipid phase. Skin excised from pig ears was mounted in Franz diffusion cells containing phosphate buffer $\mathrm{pH} 7.4$ as receptor solution, and the surface of the skin was treated with $1 \mathrm{~mL}$ sample of Nile red-loaded SLN or 
NLC dispersion. The Franz diffusion cells were kept in a water bath at $37^{\circ} \mathrm{C}$ and, after $2 \mathrm{~h}$ contact, the remaining formulation was removed and tape stripping was performed on the skin. Fifteen tapes (Scotch ${ }^{\circledR}$ packaging tape) of $2 \times 2 \mathrm{~cm}$ were used, weighting each tape before and after skin application. Nile red was extracted from the tapes, stirring with a volume of methanol during $24 \mathrm{~h}$. Tapes 1 to 5 were extracted individually and subsequent tapes were extracted in clusters (6 - 10 and 11 - 15). The extracts were filtered and Nile red was quantified by spectrophotometry UV/Vis $(\lambda=555 \mathrm{~nm})$ (Varian Cary ${ }^{\circledR} 50 \mathrm{UV}$-Vis spectrophotometer, Australia). Penetration distance was determined from tapes weight data, using Equation (3):

$$
\text { Penetration distance }=\frac{T W_{f}-T W_{0}}{S C D \times A}
$$

where $T W f$ is the tape weight after the stripping, $T W_{0}$ is the tape weight before the stripping, $S C D$ is the SC density considered as $1 \mathrm{~g} \mathrm{~cm}^{3}$ and $A$ is the tape area.

\subsection{Statistical Analysis}

The statistical analysis was performed with $\mathrm{p}<0.05$ as level of significance (STATGRAPHICS ${ }^{\circledR}$ Centurion XVI).

\section{Results and Discussion}

\subsection{Lipid Nanoparticle Characterization}

Although high pressure homogenization (HPH) is the most used technique for lipid nanoparticle preparation [9], high shear rate homogenization is a feasible technique to prepare lipid nanoparticles at laboratory scale since it is an easy handle technique, that requires low cost equipment compared to HPH, and that allows to obtain particles of nanometric size ( $200 \mathrm{~nm}$ ) [20]. Table 1 shows the results of SLN and NLC characterization.

No differences in particle size between SLN and NLC ( $p>0.05)$ were found. SLN and NLC of similar particle size were desirable so that this variable was not a factor directly influencing the results observed on the skin (occlusive effect and penetration enhancement ability). PI indicates the width of the particle size distribution. Theoretically, monodisperse distributions are described as PI $=0$, however, $\mathrm{PI}<0.2$ is considered as narrow size distribution [16]. PI $>0.2$ for the lipid nanoparticles prepared could be due to the preparation method used, giving a wider size distribution compared to HPH [20]. Zeta potential is a parameter used to predict stability of colloidal suspensions throughout storage time. A zeta potential higher than $\pm 30 \mathrm{mV}$ may assure good physical stability, being optimal when zeta potential is close to $\pm 60 \mathrm{mV}$ [21]. It is well known that zeta potential depends on the nature of the particles and the medium composition. For lipid nanoparticles, the molecular arrangement of lipids in the external layer of nanoparticles and its interaction with stabilizers are determinant. For the lipid nanoparticles prepared, poloxamer 188 was used as stabilizer, and due to its non-ionic nature, this molecule does not contribute with additional charges to zeta potential. Furthermore, the lipid that composes SLN is in fact a blend of acylglycerols (Figure 1(a)): glyceryl tribehenate (28\% - 32\%), glyceryl dibehenate (52\% - 54\%) and glyceryl monobehenate (12\% - 18\%) [22], all of them being glycerol esters of longchain-length fatty acids (C22) so that they provide neither charge nor polarity that contributes to zeta potential. On the other hand, NLC are made of the same lipid blend but including a certain amount of caprylic/capric triglyceride, which is a diacylglycerol of medium-chain-length fatty acids (Figure 1(b)). In this case, due to the non-esterified hydroxyl group of the glycerol and the length of the fatty acids, this molecule exhibits certain polarity that contributes to zeta potential, which explains the higher value compared to SLN. A similar result was observed by Teeranachaideekul et al. [18] for nanoparticles composed of cetyl palmitate and mediumchain-length triglycerides. The authors explain that it might be due to the accumulation of oil at the surface of NLC. Being the melting point of the solid lipid higher than that of the oil, when preparing NLC, the solid lipid recrystallizes first, holding a portion of the oil within the solid lipid matrix. Subsequently, the excess of oil remains in the outer shell of nanoparticles, then the oil contributes largely to zeta potential. Despite this difference in zeta potential between SLN and NLC, both values are under the desired $\pm 30 \mathrm{mV}$, which may indicate physical stability issues during storage. Nevertheless, poloxamer 188 stabilizes lipid nanoparticles because of the steric effect it produces which avoids coalescence of the particles [21]. Such steric effect is possible due to the structure and conformation of poloxamer. Poloxamer 188 is a block copolymer of ethylene 


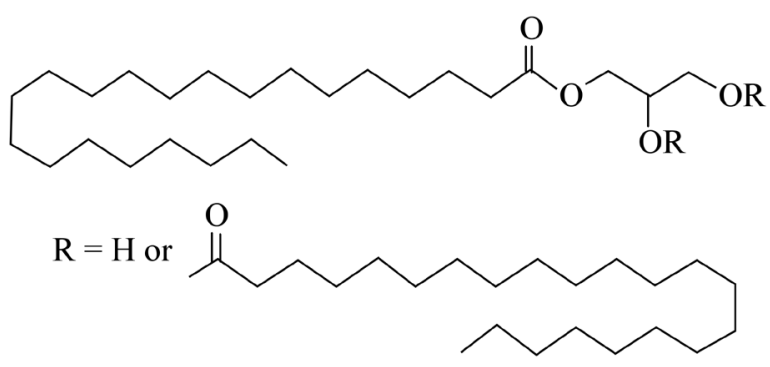

(a)

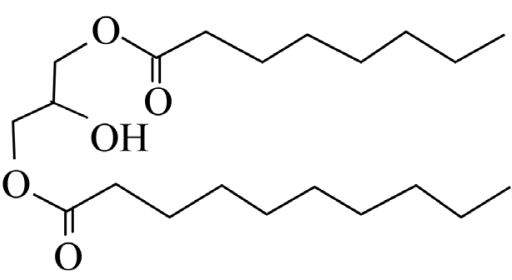

(b)

Figure 1. Chemical structure of (a) glyceryl dibehenate as a mix of glyceryl tribehenate (28\% - 32\%), glyceryl dibehenate (52\% - 54\%) and glyceryl monobehenate (12\% - 18\%); and (b) caprylic capric triglyceride.

Table 1. Particle size, PI and zeta potential of SLN and NLC prepared by high shear homogenization. Results are displayed as mean $\pm \operatorname{SD}(n=5)$.

\begin{tabular}{|c|c|c|c|}
\hline & Particle size (nm) & PI & Zeta potential (mV) \\
\hline SLN & $200 \pm 6$ & $0.436 \pm 0.022$ & $-7.5 \pm 0.6$ \\
\hline NLC $(90: 10)^{1}$ & $192 \pm 11$ & $0.311 \pm 0.014$ & $-11.1 \pm 0.8$ \\
\hline
\end{tabular}

${ }^{1}$ Glyceryl dibehenate: caprylic capric triglycerides.

oxide and propylene oxide with the formula $\mathrm{HO}\left(\mathrm{C}_{2} \mathrm{H}_{4} \mathrm{O}\right)_{80}\left(\mathrm{C}_{3} \mathrm{H}_{6} \mathrm{O}\right)_{27}\left(\mathrm{C}_{2} \mathrm{H}_{4} \mathrm{O}\right)_{80} \mathrm{H}$. The polyoxyethylene segments are hydrophilic and the polyoxypropylene segment is hydrophobic [22], so this last part of the molecule is located on the lipid nanoparticle surface whereas the long hydrophilic polymer chains are oriented towards the medium. Regarding the morphology of the lipid nanoparticles, Figure 2 shows SEM images for SLN and NLC. Both systems exhibit similar characteristics since they are assembled out of the same main lipid and were prepared under the same conditions. Particles appear as spherical or almost spherical structures of approximately $200 \mathrm{~nm}$, which agrees with the results obtained from the photon correlation spectroscopy technique.

\subsection{SLN and NLC Stability}

Physical stability of SLN and NLC was assessed measuring particle size, PI and zeta potential to determine whether there are changes or not, depending on the storage temperature and storage time. Results showed no significant changes in either PI or zeta potential through storage time under different storage conditions (data not shown). Conversely, both storage time and temperature have effect on the particle size of the lipid carriers (Figure 3). For SLN, particle size increased at 90 days and $40^{\circ} \mathrm{C}$, whereas NLC size increased at 90 days for both 25 and $40^{\circ} \mathrm{C}$ (ANOVA test showed significant differences between $40^{\circ} \mathrm{C}$ and the other two temperatures for SLN and between $5^{\circ} \mathrm{C}$ and the other two temperatures for NLC). Hence, storage at $5^{\circ} \mathrm{C}$ seems to preserve the particle size more effectively for both SLN and NLC up to 90 days.

\subsection{Test of in Vitro Occlusive Effect}

Results of in vitro occlusive effect (Figure 4) showed that occlusion factor (Equation (1)) took values between 35.9 and 38.9 throughout the test for SLN and NLC. Although no statistical difference was found between SLN and NLC, differences between $6 \mathrm{~h}$ and the other two times were found, which might be due to the formation of a film on the filter paper at the beginning of the test. When a nanoparticle suspension is applied onto the skin, lipid nanoparticles tend to fusion forming a film on the skin [23], so, it is expected that this effect occurs on the filter paper as well. The formation of this nanoparticle film was confirmed by scanning electron microscopy. Figure 5 shows the presence and characteristics of the film formed with the lipid carriers: fibers that compose filter paper (without treatment) are shown in Figure 5(a), whereas Figure 5(b) and Figure 5(c) show these fibers covered by the lipid carriers. This film is thought to be responsible for the occlusive effect observed in the test, as it acts like a barrier that avoids water vapor to escape through the filter paper. As shown, the films formed by both SLN and NLC are alike between each other. It has been demonstrated previously that the degree 

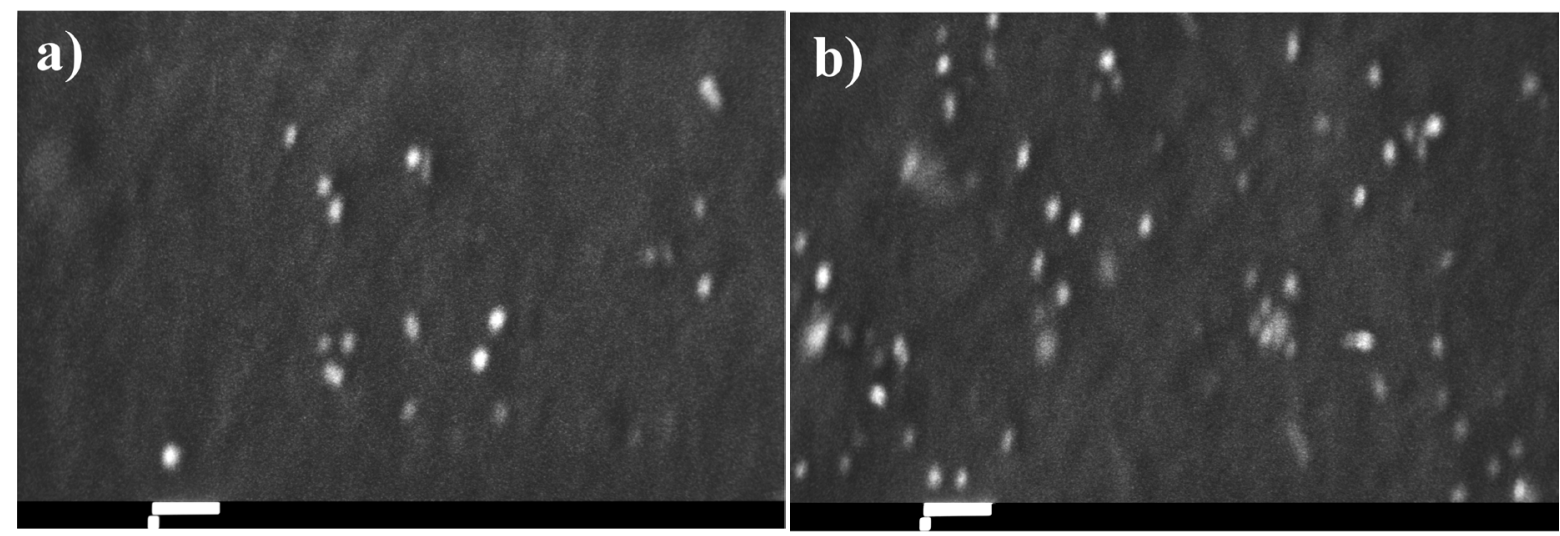

Figure 2. SEM images of a) SLN and b) NLC. The bar at the bottom equals $1 \mu \mathrm{m}(10,000 \times)$.

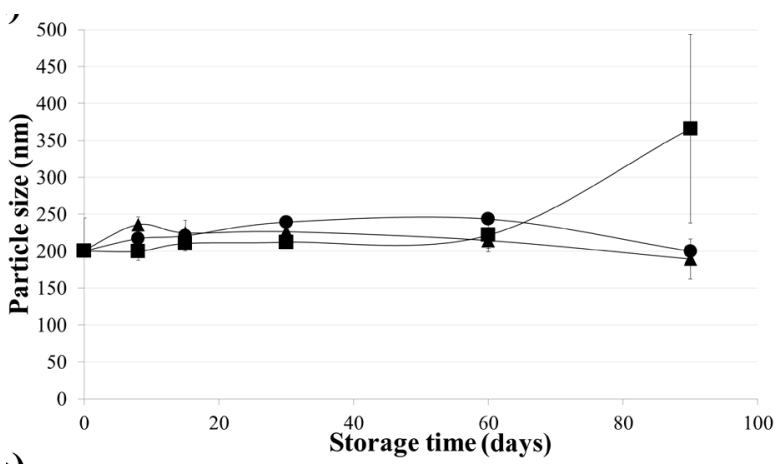

(a)

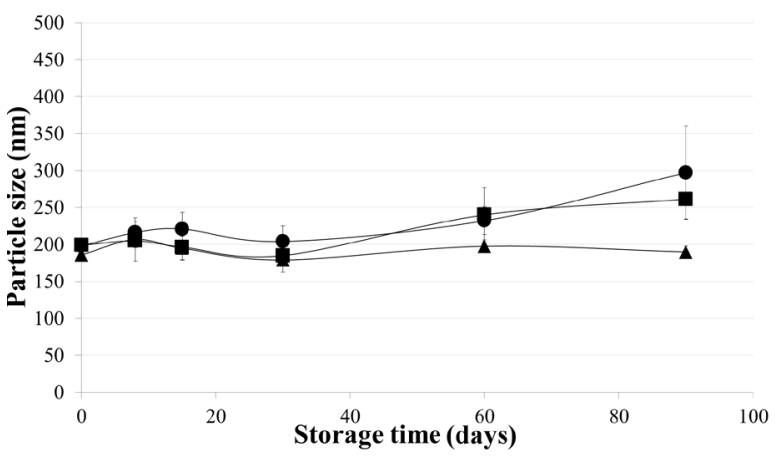

(b)

Figure 3. Lipid nanoparticle stability at different temperatures: $(\boldsymbol{\Delta}) 5^{\circ} \mathrm{C},(\boldsymbol{O}) 25^{\circ} \mathrm{C}$, (ם) $40^{\circ} \mathrm{C}$. Particle size of (a) SLN and (b) NLC plotted as a function of time $(0,8,15,30,60$ and 90 days). Values are plotted as mean $(\mathrm{n}=3)$ and the bars correspond to SD. Significant differences were found between $40^{\circ} \mathrm{C}$ and the other two temperatures for SLN and between $5^{\circ} \mathrm{C}$ and the other two temperatures (NLC).

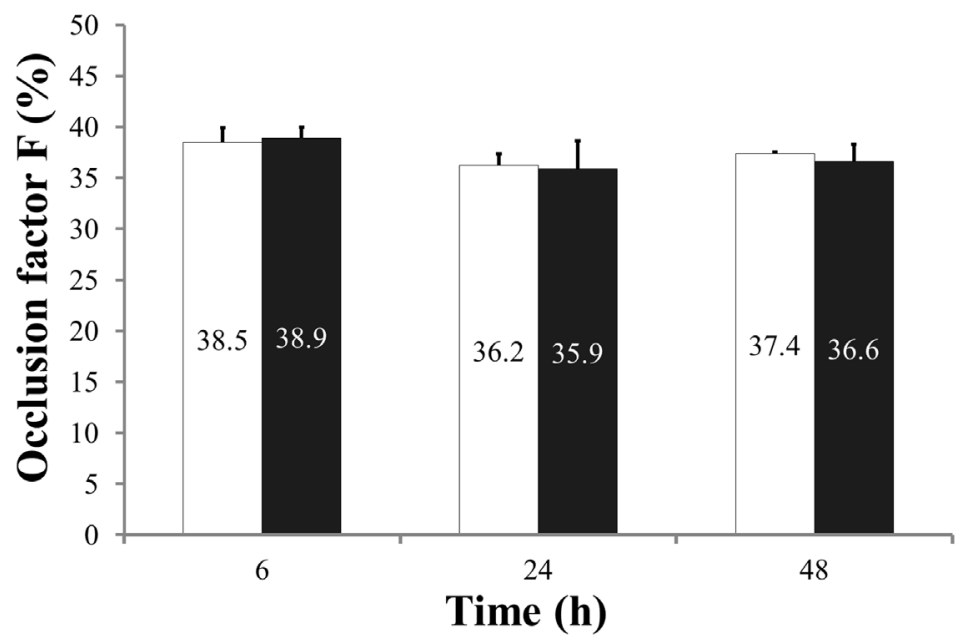

Figure 4. Occlusion factor (F) for SLN ( $\square$ ) and NLC ( $\square$ ) at 6, 24 and 48 h. Data is given as the mean value and the bars correspond to SD.

of occlusion of these films depends on the particle size [24], therefore, as the size of SLN and NLC are very close, it is clear that the inclusion of oil for preparing NLC does not have effect either on the film formation or on the occlusive effect. 


\subsection{Effect of SLN and NLC on the TEWL}

In order to evaluate the effect of SLN and NLC on the TEWL value, reduction of this parameter as percentage (Equation (2)) is shown in Figure 6. A significant reduction in TEWL of intact skin was observed for SLN and NLC respectively, without statistical difference between these systems $(p>0.05)$. Such effect is due to the occlusive effect of lipid carriers as result of film formation on the skin. The film formed at the surface of pig skin was observed by SEM. Figure 7(a) shows intact skin without treatment, where the microrelief lines as well
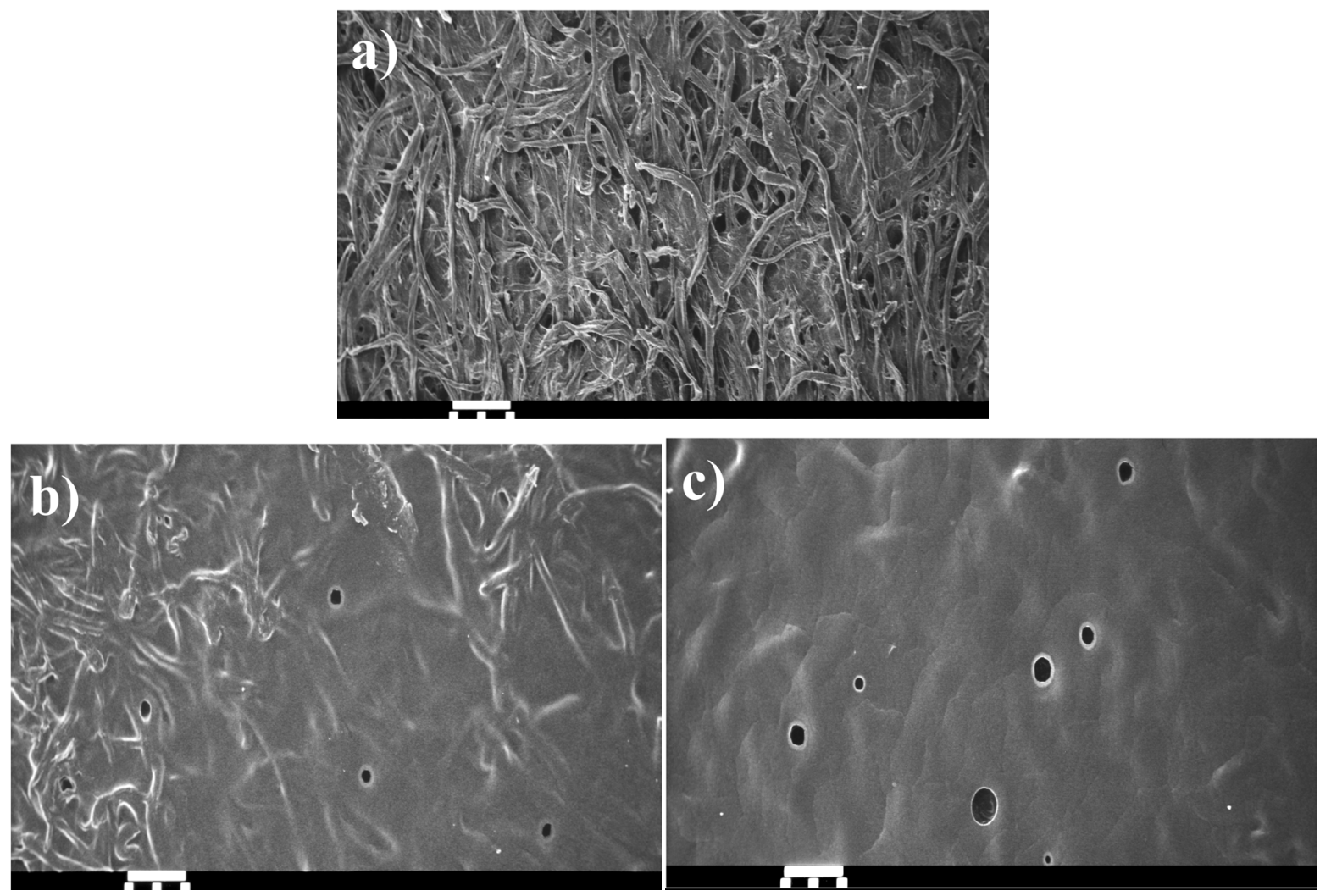

Figure 5. SEM images showing filter paper: a) without lipid nanoparticles; b) with SLN; c) with NLC. Bar at the bottom equals $100 \mu \mathrm{m}(100 \times)$.

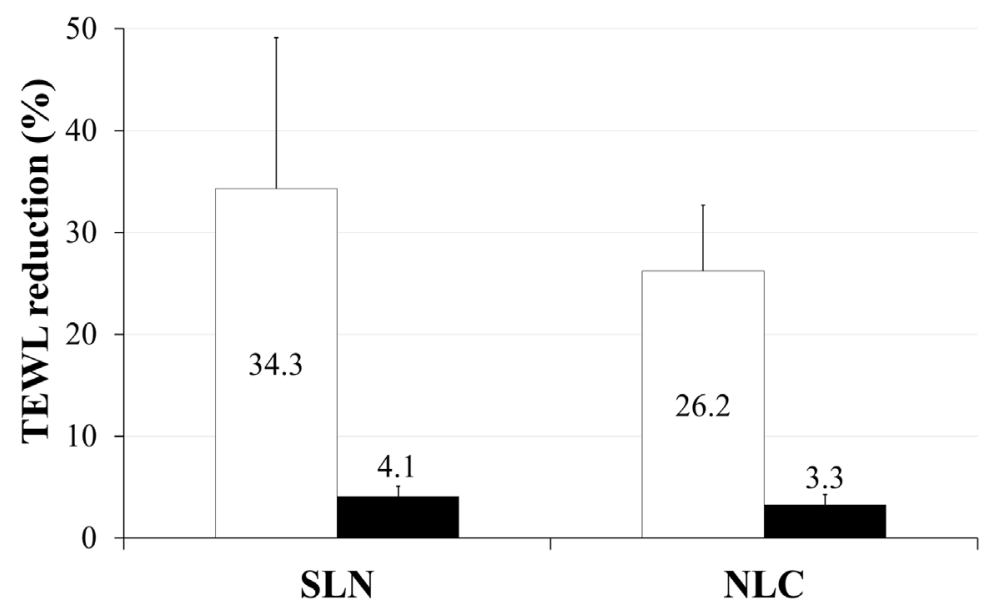

Figure 6. Reduction of the TEWL after treatment with SLN and NLC in intact ( $\square$ ) and impaired skin ( $\square$ ). Values are plotted as mean $(n=6)$ and the bars correspond to SD. 
as hairs are clearly seen. On the contrary, in Figure 7(b) and Figure 7(c), which correspond to intact skin treated with SLN and NLC respectively, microrelief lines and some parts of hair remain under the film formed by the carriers. In the case of impaired skin, carriers did not have a significant effect on the TEWL ( $p>0.05$ ). Due to stripping of SC the high humidity on the skin surface prevents film formation, since lipid carrier suspensions applied on the skin have to lose their own humidity in order to form the film. Jenning et al. [25] studied water loss from SLN, by applying lipid nanoparticles on a glass surface and measuring water loss. They determined that formulation lost their own water almost completely within $1 \mathrm{~h}$. In the present work, even $2 \mathrm{~h}$ after applying formulations, the film was not formed on the stripped-skin surface and therefore, a reduction in TEWL was not detected.

\subsection{Penetration Test through Pig Skin}

Tape stripping is considered a useful non-invasive technique that when coupled with other techniques, it can provide information about the transport of substances through the SC [11]. In this work, tape stripping was used to determine the amount as well as the penetration depth (Equation (3)) through the SC of Nile red included in both SLN and NLC. Lipid nanoparticles are unable to penetrate through human skin because of their size, but

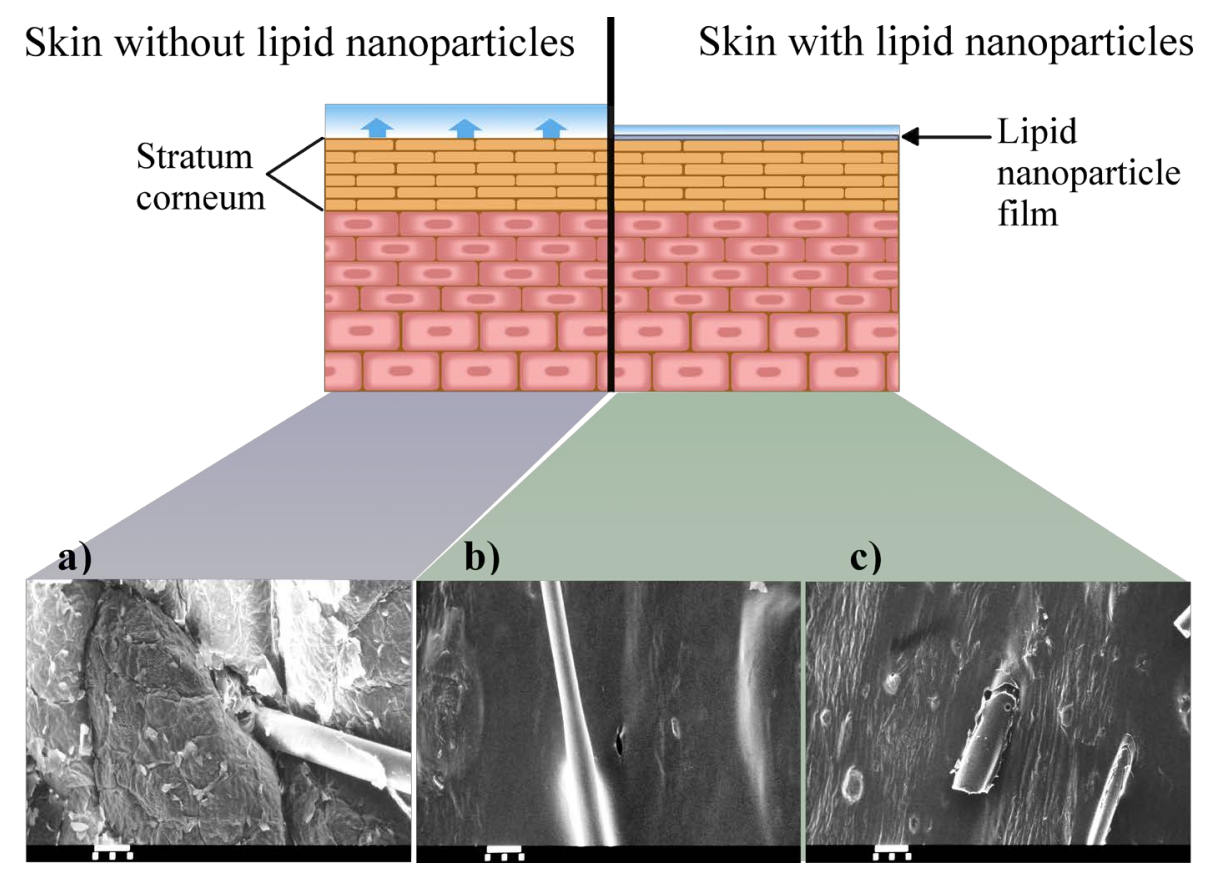

Figure 7. SEM images showing intact pig skin: a) without lipid nanoparticles; b) with SLN; c) with NLC. Bar at the bottom equals $100 \mu \mathrm{m}(100 \times)$.

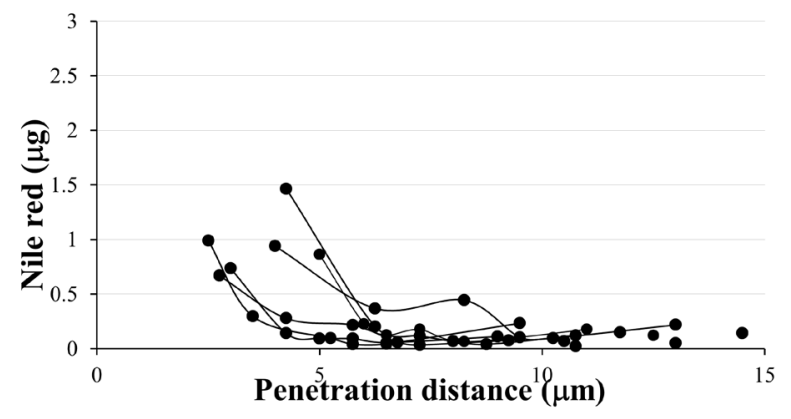

(a)

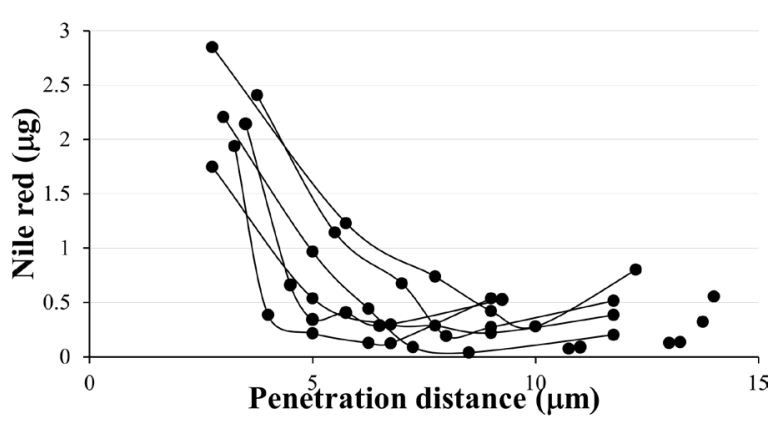

(b)

Figure 8. Penetration profile of Nile red throughout the stratum corneum. The graph shows the amount of Nile red versus penetration distance for a) SLN and b) NLC $(n=6)$. 
they can help actives to reach deeper skin layers due to the interaction of nanoparticle lipids with the extracellular lipid matrix of the skin. Figure 8 shows the penetration profile of Nile red included in both SLN and NLC, being NLC the system that favors the penetration of a greater amount of Nile red. At a distance of $12.2 \pm$ $1.5 \mu \mathrm{m}(\mathrm{SLN})$ and $12.6 \pm 1.4 \mu \mathrm{m}$ (NLC), the total amount of Nile red penetrated was $1.7 \pm 0.4 \mu \mathrm{g}$ and $4.7 \pm 1.3$ $\mu \mathrm{g}$, respectively. This means that, even if a similar occlusive effect was found for both systems, the amount of Nile red penetrated was 2.7 fold higher for the NLC. This fact suggests that lipid nanocarrier composition affects the ability to enhance penetration of molecules through the SC, due to differences in the interaction of lipids with skin components. It has been reported that NLC have the ability to get into deeper layers of the SC. Penetration through pig skin of flufenamic acid (a model drug) included in SLN and NLC, was monitored using tape stripping. Researchers found that NLC showed the deepest skin penetration [12]. In other study, the penetration enhancement ability of lipid nanoparticles was related to the solubility parameter (SP) of lipids [26]. SP is useful to predict the release and skin penetration of any molecule by its interaction with skin lipids. Components with a SP close to that of the skin may have good miscibility with the skin lipids, and thus, can lead to greater penetration. As reported by Jensen et al. [26], pig skin SP is estimated in 10, thus lipids with a SP value nearby 10 are expected to exhibit a better interaction with skin lipids and so, the penetration is expected to be greater as well. In this work, SP of glyceryl behenate and caprylic/capric triglyceride was calculated upon the Fedors substituents method [27]. SP values are estimated to be 9.34 for glyceryl behenate and 10.36 for caprylic/ capric triglyceride, therefore, the inclusion of this oil may improve the interaction of NLC with skin lipids as well as the penetration enhancement ability through the SC, since triglycerides are more miscible with skin lipids for having a closer value to the skin SP. Moreover, partition of Nile red within NLC contributes to explain its greater penetration. Nile red distribution within NLC was studied by fluorescence spectroscopy [28]. The authors found that the fluid lipid phase of NLC composed of glyceryl behenate and caprylic/capric triglyceride was enriched in Nile red ( $65 \% \pm 8 \%$ ). Hence, a better interaction with skin lipids is expected, allowing Nile red to penetrate into deeper SC layers when it is included in NLC.

\section{Conclusion}

The occlusive effect and penetration enhancement ability of SLN and NLC were studied in order to determine differences between these carrier systems. According to the tests performed, both SLN and NLC produced an occlusive effect of similar magnitude, which is due to the ability of lipid nanoparticles to form a film. No differences were found between film appearance and the occlusive effect degree produced by both SLN and NLC, suggesting that differences in composition did not affect those parameters. In contrast, neither occlusive effect nor film formation was observed in impaired skin, because SC removal led to an increased TEWL and so a higher humidity on the skin surface, which avoided film to form and to produce occlusion. Regarding the penetration enhancement ability of lipid carriers, Nile red included in NLC penetrated into deeper SC layers compared to dye included in SLN. Enhanced penetration is attributed to the influence of particle composition and its interaction with SC components. These findings suggest that NLC may be useful to enhance the penetration of some cosmetic ingredients into deep layers of the stratum corneum.

\section{Acknowledgements}

The authors acknowledge funding from CONACYT (129320, 271124) and PAPIIT (IN216313). The authors also thank to Mr. Rodolfo Robles Gómez for the support to obtain microscope images.

\section{References}

[1] Müller, R.H., Radteke, M. and Wissing, S.A. (2002) Solid Lipid Nanoparticles (SLN) and Nanostructured Lipid Carriers (NLC) in Cosmetic and Dermatological Preparations. Advanced Drug Delivery Reviews, 54, S131-S155. http://dx.doi.org/10.1016/S0169-409X(02)00118-7

[2] Jores, K., Mehnert, W., Drechsler, M., Bunjes, H., Johann, C. and Mäder, K. (2004) Investigations on the Structure of Solid Lipid Nanoparticles (SLN) and Oil-Loaded Solid Lipid Nanoparticles by Photon Correlation Spectroscopy, Field-Flow Fractionation and Transmission Electron Microscopy. Journal of Controlled Release, 95, 217-227. http://dx.doi.org/10.1016/j.jconrel.2003.11.012

[3] Teeranachaideekul, V., Müller, R.H. and Junyaprasert, V.B. (2007) Encapsulation of Ascorbyl Palmitate in Nanostructured Lipid Carriers (NLC) -Effects of Formulation Parameters on Physicochemical Stability. International Journal of Pharmaceutics, 340, 198-206. http://dx.doi.org/10.1016/j.ijpharm.2007.03.022 
[4] Contri, R.V., Fiel, L.A., Pohlman, A.R., Guterres, S.S. and Beck, R.C.R. (2011) Transport of Substances and Nanoparticles across the Skin and in Vitro Models to Evaluate Skin Permeation and/or Penetration. In: Beck, R., Guterres, S. and Pohlman, A., Eds., Nanocosmetics and Nanomedicines, New Approaches for Skin Care, Springer, Berlin, 3-35. http://dx.doi.org/10.1007/978-3-642-19792-5_1

[5] Puglia, C., Damiani, E., Offerta, A., Rizza, L., Tirendi, G.G., Tarico, M.S., Curreri, S., Bonina, F. and Perrotta, R.E. (2014) Evaluation of Nanostructured Lipid Carriers (NLC) and Nanoemulsions as Carriers for UV-Filters: Characterization, in Vitro Penetration and Photostability Studies. European Journal of Pharmaceutical Sciences, 51, 211-217. http://dx.doi.org/10.1016/j.ejps.2013.09.023

[6] Souto, E.B. and Müller, R.H. (2008) Cosmetic Features and Application of Lipid Nanoparticles $\left(\operatorname{SLN}^{\circledR}\right.$, NLC $\left.^{\circledR}\right)$. International Journal of Cosmetic Science, 30, 157-165. http://dx.doi.org/10.1111/j.1468-2494.2008.00433.x

[7] Wissing, S., Lippacher, A. and Müller, R. (2001) Investigations on the Occlusive Properties of Solid Lipid Nanoparticles (SLN). Journal of Cosmetic Science, 52, 313-324.

[8] Wissing, S. and Müller, R. (2003) The Influence of Solid Lipid Nanoparticles on Skin Hydration and ViscoelasticityIn Vivo Study. European Journal of Pharmaceutics and Biopharmaceutics, 56, 67-72. http://dx.doi.org/10.1016/S0939-6411(03)00040-7

[9] Pardeike, J., Hommoss, A. and Müller, R. (2009) Lipid Nanoparticles (SLN, NLC) in Cosmetic and Pharmaceutical Dermal Products. International Journal of Pharmaceutics, 366, 170-184. http://dx.doi.org/10.1016/j.ijpharm.2008.10.003

[10] Müller, R., Petersen, R., Hommoss, A. and Pardeike, J. (2007) Nanostructured Lipid Carriers (NLC) in Cosmetic Dermal Products. Advanced Drug Delivery Reviews, 59, 522-530. http://dx.doi.org/10.1016/j.addr.2007.04.012

[11] Iannuccelli, V., Coppi, G., Romagnolli, M., Sergi, S. and Leo, E. (2013) In Vivo Detection of Lipid-Based Nano- and Microparticles in the Outermost Human Stratum Corneum by EDX Analysis. International Journal of Pharmaceutics, 447, 204-212. http://dx.doi.org/10.1016/j.ijpharm.2013.03.002

[12] Schwarz, J., Weixelbaum, A., Pagitsch, E., Löw, M., Resch, G. and Valenta, C. (2012) Nanocarriers for Dermal Drug Delivery: Influence of Preparation Method, Carrier Type and Rheological Properties. International Journal of Pharmaceutics, 437, 83-88. http://dx.doi.org/10.1016/j.ijpharm.2012.08.003

[13] Montenegro, L., Sinico, C., Castangia, I., Carbone, C. and Puglisi, G. (2012) Idebenone-Loaded Solid Lipid Nanoparticles for Drug Delivery to the Skin: In Vitro Evaluation. International Journal of Pharmaceutics, 434, 169-174. http://dx.doi.org/10.1016/j.ijpharm.2012.05.046

[14] Tiwari, R. and Pathak, K. (2011) Nanostructured Lipid Carrier Versus Solid Lipid Nanoparticles of Simvastatin: Comparative Analysis of Characteristics, Pharmacokinetics and Tissue Uptake. International Journal of Pharmaceutics, 415, 232-243. http://dx.doi.org/10.1016/j.ijpharm.2011.05.044

[15] Kovacevic, A., Savic, S., Vuleta, G., Müller, R. and Keck, C. (2011) Polyhydroxy Surfactants for the Formulation of Lipid Nanoparticles (SLN and NLC): Effects on Size, Physical Stability and Particle Matrix Structure. International Journal of Pharmaceutics, 406, 163-172. http://dx.doi.org/10.1016/j.ijpharm.2010.12.036

[16] Das, S., Kiong, W. and Tan, R. (2012) Are Nanostructured Lipid Carriers (NLCs) Better Than Solid Lipid Nanoparticles (SLNs): Development, Characterizations and Comparative Evaluations of Clotrimazole-Loaded SLNs and NLCs? European Journal of Pharmaceutical Sciences, 47, 139-151. http://dx.doi.org/10.1016/j.ejps.2012.05.010

[17] Bose, S. and Michniak-Kohn, B. (2013) Preparation and Characterization of Lipid Based Nanosystems for Topical Delivery of Quercetin. European Journal of Pharmaceutical Sciences, 48, 442-452. http://dx.doi.org/10.1016/j.ejps.2012.12.005

[18] Teeranachaideekul, V., Boonme, P., Souto, E.B., Müller, R.H. and Junyaprasert ,V.B. (2008) Influence of Oil Content on Physicochemical Properties and Skin Distribution of Nile Red-Loaded NLC. Journal of Controlled Release, 128, 134-141. http://dx.doi.org/10.1016/j.jconrel.2008.02.011

[19] Levin, J. and Maibach, H. (2009) The Correlation between Transepidermal Water Loss and Percutaneous Absorption: An Overview. In: Barel, A., Paye, M. and Maibach, H. Eds, Handbook of Cosmetic Science and Technology, Informa Healthcare, NewYork, 165-171. http://dx.doi.org/10.1201/9780849359033.ch43

[20] Mehnert, W. and Mäder, K. (2001) Solid Lipid Nanoparticles: Production, Characterization and Applications. Advanced Drug Delivery Reviews, 47, 165-196. http://dx.doi.org/10.1016/S0169-409X(01)00105-3

[21] Garzón, M., Vázquez, M., Villafuerte, L., García, B. and Hernández, A. (2009) Effect of Formulation Components on the Properties of Solid Lipid Nanoparticles. Revista Mexicana De Ciencias Farmacéuticas, 2, 26-40.

[22] Rowe, R., Sheskey, P. and Owen, S. (2006) Handbook of Pharmaceutical Excipients. Pharmaceutical Press, London, 304-305, 535-538.

[23] Wissing, S. and Müller, R. (2001) A Novel Sunscreen System Based on Tocopherol Acetate Incorporated into Solid Lipid Nanoparticles. International Journal of Cosmetic Science, 23, 233-243. 
http://dx.doi.org/10.1046/j.1467-2494.2001.00087.x

[24] Wissing, S. and Müller, R. (2003) Cosmetic Applications for Solid Lipid Nanoparticles (SLN). International Journal of Pharmaceutics, 254, 65-68. http://dx.doi.org/10.1016/S0378-5173(02)00684-1

[25] Jenning, V., Gysler, A., Schäfer-Korting, M. and Gohla, S. (2000) Vitamin A Loaded Solid Lipid Nanoparticles for Topical Use: Occlusive Properties and Drug Targeting to the Upper Skin. European Journal of Pharmaceutics and Biopharmaceutics, 49, 211-218. http://dx.doi.org/10.1016/S0939-6411(99)00075-2

[26] Jensen, L., Petersson, K. and Nielsen, H. (2011) In Vitro Penetration Properties of Solid Lipid Nanoparticles in Intact and Barrier-Impaired Skin. European Journal of Pharmaceutics and Biopharmaceutics, 79, 68-75. http://dx.doi.org/10.1016/j.ejpb.2011.05.012

[27] James, K.C. (1986) Solubility and Related Properties. Marcel Dekker, New York.

[28] Lombardi, S., Regehly, M., Sivaramakrishnan, R., Mehnert, W., Korting, H.C., Danker, K., Röder, B., Kramer, K.D. and Schäfer-Korting, M. (2005) Lipid Nanoparticles for Skin Penetration Enhancement-Correlation to Drug Localization within the Particle Matrix as Determined by Fluorescence and Parelectric Spectroscopy. Journal of Controlled Release, 110, 151-163. http://dx.doi.org/10.1016/j.jconrel.2005.09.045 